\title{
ENVEJECIMIENTO Y CAMPO DE LA EDAD: ELEMENTOS SOBRE LA PERTINENCIA DEL CONOCIMIENTO GERONTOLOGICO*
}

\author{
EUGENIO GUTIÉRREZ V.** \\ PATRICIO RÍOS S.***
}

\begin{abstract}
RESUMEN
El texto que se presenta a continuación, expone los componentes de una teoría de la producción de las edades. Siguiendo las modulaciones de Pierre Bourdieu, el texto da los fundamentos del campo de la edad, expone los subcampos en los cuales se apoya, así como bosqueja los agentes que operan en el campo de la edad. El artículo postula esta construcción como el fundamento del objeto de estudio de la gerontología social.
\end{abstract}

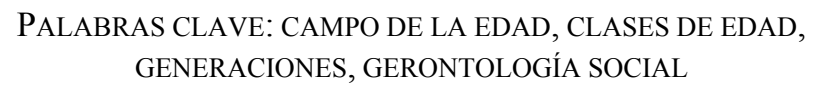

* Documento producido en el marco del desarrollo del proyecto ConicytBanco Mundial, Anillos en Ciencias Sociales, ACS-33 «Observatorio Social del Envejecimiento y la Vejez en Chile».

** Antropólogo Social, Investigador Asociado del Observatorio Social del Envejecimiento y la Vejez en Chile y de la Corporación AÑos. E-Mail: corpan@vtr.net.

*** M. A. Literatura, Investigador Titular del Observatorio Social del Envejecimiento y la Vejez en Chile y de la Corporación AÑos. E-Mail: corpan@vtr.net. 


\section{RESUMO}

\section{ENVELHECIMENTO E CAMPO DE IDADE: ELEMENTOS SOBRE} A PERTINÊNCIA DO CONHECIMENTO GERONTOLÓGICO

O texto apresentado a seguir expõe os componentes de uma teoria da produção das idades. Seguindo as modulações de Pierre Bourdieu, o texto dá os fundamentos do campo de idade, expõe os subcampos sobre os quais se sustenta, bem como esboça os agentes que operam no campo de idade. $\mathrm{O}$ artigo pressupõe esta construção como o fundamento do objeto de estudo da gerontologia social.

Palavras chave: CAmpo de idade, Classes de idade, GERAÇÕEs, GERONTOLOGIA SOCIAL

\section{Abstract \\ OLDNESS AND AGE FIELD: ELEMENTS OVER THE RELEVANCY OF THE GERONTOLOGICAL KNOWLEDGE}

The following text, displays the components of theory the production of ages. Following Pierre Bordieu modulations, the text submits the foundations of the age field, exposes the sub fields on which it supports and designs the agents acting in the age field. The article postulates this construction as the foundation of the social gerontological study.

Key WORdS: Age FIELD, Kinds OF AGE, GENERATIONS, SOCIAL GERONTOLOGY 


\section{INTRODUCCIÓN}

EN CUANTO CATEGORÍA SOCIAL o cultural, la edad ha constituido, (i) desde el punto de vista de la antropología, junto con el sexo, uno de los principios en los que se fundamenta la organización social; ii) en la literatura sociológica, una variable interviniente en la realidad social que marca diferencias; iii) en el estudio de las poblaciones, una variable central en su perspectiva analítica; iv) en la vida cotidiana, un antecedente de inclusión o de exclusión social, entre otros efectos.

Hay disciplinas, sin embargo, en las que la edad se transforma en un componente constitutivo de su objeto de estudio. Así, la psicología evolutiva o disciplinas médicas. También la gerontología social.

No obstante, sus fuentes inspiradoras de orientación en las ramas del conocimiento no han sido precisamente disciplinas, desarrollos ligados al eje de las edades, sino, más bien, la gerontología social de los últimos cincuenta años, en su vertiente más tradicional, continúa sin poder desligarse de dos factores que oscurecen sus fronteras. Estos factores se refieren a: i) su dependencia y/o subordinación/identificación con la gerontología o geriatría, disciplina médica de la última edad; ii) un descriptivismo disperso y oscilante entre lo apocalíptico y lo didáctico. ${ }^{1}$

Es obvio, además, tener que sumar a este estado de la situación la constatación de la pobreza de atención y de reflexión que a nivel de la cultura ha recibido la categoría edad. Variable del funcionamiento social o fundamento de la división del trabajo en la observación de las

1 Una buena parte de los textos tradicionales destinados a presentar los alcances de la Gerontología en cuanto disciplina coinciden en: i) no asignarle un objeto de estudio unitario, por el contrario, insisten en referir sus intereses disciplinarios a parcelas de ciencias vinculadas, por una parte al envejecimiento - teorías biológicas y psicológicas del envejecimiento - y por otra, a la vejez, una de las edades componentes del ciclo vital; ii) asignarle un carácter multidisciplinario; iii) constatar el empleo de metodologías provenientes de la sicología, la sociología, la antropología, la historia, la demografía, etc.; iv) por lo anterior, mantener una vacilación últimamente menos extendida entre Gerontología, a secas, Geriatría y Gerontología Social. Vale la pena, entonces visualizar que la Gerontología Social se ha instalado en un campo de fronteras disciplinarias difuso y en el cual la edad, la naturaleza etaria de su objeto de estudio aparece compartido y hasta opacado por el componente del envejecimiento. 
sociedades menos complejas, la categoría edad ha sido desplegada básicamente por las ciencias de la conducta derivando en un abanico de edades o etapas del desarrollo humano, cada una de ellas caracterizada por un conjunto de rasgos compartidos por el conjunto de los individuos situados en los rangos etarios asignados a la etapa de la cual se trate: niñez, adolescencia, juventud, etc.

En esta mirada, la lectura de las edades ha sido abordada en la relación que guarda el paso del tiempo —continuum segmentado en años- con cambios biológicos asociados, a su vez, con comportamientos y capacidades recurrentes, a la manera de quien observa, ahistóricamente, los efectos del paso del tiempo en los ejemplares de una especie biológica dada.

Este formato de observación lleva al convencimiento que un individuo de 60 años de edad en Nueva York y en Tel Aviv poseen sentimientos, nociones, creencias, actitudes, lecturas y prácticas de las relaciones familiares, habilidades, capacidades, percepciones del presente y del futuro, etc., muy próximos, en la medida que la edad que se tiene es la misma.

Más de una evidencia existe en el sentido de que no hay nada más alejado de una certeza que la homologación de los sujetos de acuerdo a sus edades cronológicas. Envejecer en una sociedad organizada estructuralmente para la guerra o en otra organizada para el consumo producirá sin lugar a dudas ethos diferentes entre los individuos que comparten una misma edad.

La delegación que la cultura dominante ha realizado para reflexionar la edad en las ciencias del comportamiento escamotea el punto de partida más elemental y más obvio que es necesario observar cuando se comienza a reflexionar sobre la edad: las distintas edades son distinguibles en el eje de la reproducción/distinción progenitores (mayores)/descendientes (menores) y en el eje del poder, en el cual pueden ventilarse las distinciones etarias de acuerdo a cómo los mayores ejercen el poder y producen las edades.

En lo importante, en este documento, se parte de la sospecha fundada que el uso que la cultura dominante hace de la categoría «edad» no alude a nada sustancial ni universal y que para explorar esta sospecha es imprescindible adoptar un punto de vista diferente: el de la producción de las edades. ${ }^{2}$ El trabajo presente postula que el «contex-

2 La inquietud por separar la edad de la adultez mayor en una adultez mayor sana y otra enferma se encuentra con insistencia en múltiples 
to» de las «edades» representa un espacio, una referencia cultural mucho más apropiado que otros para el proyecto de una gerontología social que desde su etimología aparece adscrita al estudio de los más envejecidos, a la última de las edades antes de la muerte.

Este punto de partida pareciera ser un pilar de apoyo más prometedor que los tradicionales. La adultez mayor adquiere sentido en la medida que se instala al interior de un haz de relaciones producido históricamente con las otras edades, también históricamente producidas que se hace necesario demarcar y describir, generando las unidades y las categorías de estudio que sean del caso.

Concebida en este marco, la gerontología social va a encuadrarse en un campo cultural bien definido: el de la edad que, por una parte implica el paso del tiempo, es decir el envejecimiento y que por otra, implica a las edades y su producción. En otras palabras, no puede desligarse el paso del tiempo - el envejecer- de la construcción social y cultural que la sociedad hace de éste sin pagar el precio deformante de «naturalizar» el proceso de envejecer, de biologizarlo, falseando el carácter primariamente histórico y social que desde esta perspectiva éste posee y como consecuencia escamotear el rasgo de «fenómenos socialmente producidos» que tienen las edades. ${ }^{3}$

textos gerontológicos. No obstante, esta confusión se ha instalado con fuerza en el paradigma asistencialista que predomina en el imaginario de la vejez a nivel societal y que tiende a reproducirse una y otra vez en el sector de los servicios y de las políticas públicas cuyos destinatarios sean envejecientes mayores.

3 Por el lado de las ciencias sociales, existen referencias de disciplinas que relevan la edad como eje ordenador de sus estudios. Cabe mencionar a este respecto la llamada Antropología de las Edades (ver Feixa). Según Feixa, desde sus inicios, la antropología ha relevado la edad como categoría ordenadora del mundo social y ha producido textos clásicos a este respecto. Por ejemplo, entre otros, los trabajos de Van Gennep (1909) sobre los ritos de paso y de Mead (1929) sobre comparación intercultural de grupos de edad. Según Feixa, el interés por reflexionar la edad, pese a estos antecedentes, sin embargo, no ha sido una preocupación central de la antropología hasta la década del 80 en la que ha comenzado a emerger un cierto espacio de debate y de consolidación de investigaciones conocido como «antropología de la edad». El campo disciplinario apuntado navega entre aguas que atraviesan disciplinas vinculadas a la antropología general, tales como «la antropología física, la etología, la lingüística o la psicología» y fenómenos de frontera, entre 
El paso del tiempo, el envejecimiento, son categorías que adquieren significación al interior de un marco social y cultural determinado. En este sentido, en la sociedad moderna estas categorías son condicionadas de manera decisiva por la acción de agentes específicos - los Estados, el mercado, la sociedad civil — de la cual son productos las edades.

Los párrafos que continúan identifican la existencia de un campo llamado edad y se lo describe principalmente con la ayuda de los instrumentos conceptuales de Bourdieu, además de postularlo como un espacio cultural privilegiado desde el cual generar reflexión gerontológica.

\section{BOURDIEU: CAMPO/HABITUS, CAPITAL}

Como ha sido dicho, desde la pertinencia de la gerontología social que aquí se postula, es decir, desde su preocupación disciplinaria — dar cuenta del envejecimiento y la vejez-, tanto el envejecer como las edades, entre ellas, la vejez, se inserta en un campo acotado del funcionamiento y de la práctica social: la edad. Al interior del mismo, sin embargo, la edad se presenta también como el capital simbólico, en torno a cuya disputa, se constituye el campo de la edad.

Hay que precisar desde ya que aquí se habla de la edad como un campo social y como un capital simbólico en el sentido establecido por la escuela sociológica de Pierre Bourdieu.

Sabido es que Bourdieu concibe la sociedad como un macroespacio social constituido por una multiplicidad de campos, cada uno de ellos relativamente autónomo y dotado de sus propias convenciones de funcionamiento que son intransferibles. Cada campo se constituye a su vez a partir de la disputa entablada por los diversos agentes que

otros, en el orden cognitivo, las relaciones entre el «desarrollo somático y evolución mental, entre aprendizaje humano y animal, entre dominio del lenguaje y socialización» y en otros órdenes, las relaciones «entre ciclo vital, cultura y personalidad» (Feixa). Asimismo, apunta que en la tradición anglosajona, suele incorporarse burocráticamente a esta área, estudios sobre tercera edad, etnografía escolar, estudios sobre juventud que se asimilan a la antropología de la marginación, en tanto que los estudios sobre la adultez «brillan literalmente por su ausencia». El presente texto pretende explorar la autonomía de un campo sociocultural centrado en la edad y no necesariamente desde la perspectiva de la Antropología de las Edades. 
operan en él por la creación, el control, la posesión, el acrecentamiento y/o la reproducción de un capital específico. Metodológicamente hablando, los campos no son realidades que se presenten directamente a la observación del cientista social ni son deducibles de las conductas de los agentes sociales. Son espacios estructurados de posiciones analizables independientemente de las realidades de sus ocupantes (Bourdieu, 1969). Como toda su construcción teórica, la teorización de los campos en Bourdieu obedece a la necesidad metodológica de romper con los modos realistas o sustancialistas de pensar la vida social para reflexionarla de manera relacional (Bourdieu y Wacquant, 1995). En este sentido, los campos de Bourdieu constituyen sistemas de posiciones y de relaciones cuyas lógicas internas hay que develar. En esta tarea, Bourdieu ha dado cuenta de numerosos campos: el religioso, del Estado, de la producción cultural, del arte, etc.

Las estructuras relativamente estables que articulan el espacio social conocidas como campos en la teorización de Bourdieu se especifican en cuanto sistemas de relaciones, no sólo en función de las posiciones relativas de sus componentes internos, sino por oposición a la noción de habitus de los agentes que operan en él. Fruto de la diversidad de prácticas e intercambios sociales en los que se involucran los sujetos, agentes o instituciones al interior del conjunto de restricciones y de condiciones de los campos específicos en que suceden esos intercambios, los sujetos, agentes e instituciones incorporan habilidades, conocimientos, nociones, a la vez que capacidades adecuadas de desempeño a las estructuras de los campos, al modo de un sistema de percepciones y disposiciones estructuradas y estructurantes que orientan su accionar en la práctica. Como se ve, el habitus es a la vez el producto de las prácticas de los sujetos ejercidas en los diversos campos -interiorización de las convenciones del campo que orientan sus prácticas - , pero, simultáneamente, disposiciones para ejercer la vida práctica con la potencia suficiente como para modificar las estructuras del campo.

La oposición habitus/campo es más que una relación lógica de suposición de términos. Es una relación nacida e instaurada en la práctica por la práctica misma que divide el proceso histórico del cual forma parte en cosa ya hecha que se reconoce - campo- para su reproducción y/o cambio, fruto esto último de los habitus de los agentes que operan en los campos en un momento determinado.

Habitus y campo son, pues, por una parte, categorías analíticas que funcionan sólo en la medida que son términos que se suponen 
mutuamente, al interior de una oposición significante, y por otra, son categorías del ámbito histórico, es decir, de la racionalidad específica de los acontecimientos históricos.

Ampliando las afirmaciones realizadas más arriba y haciendo una síntesis apretada de lo que Bourdieu entiende por un campo social, es necesario referirse a los siguientes rasgos del mismo.

i) Constituye un microcosmos dentro del macrocosmos del espacio social global; ii) las reglas del juego y las apuestas que vertebran el campo refieren a reglas y apuestas específicas de ese campo y no son transferibles al de otro campo; iii) el juego interno de cada campo se instituye por la disputa que los agentes que actúan en cada campo establecen en pos de la apropiación y/o de la recomposición del capital específico y legitimado del campo; iv) un campo, asimismo, implica un sistema o un espacio estructurado de posiciones las que son ocupadas por los diversos agentes que se disputan un capital asimétricamente distribuido, factor que determina posiciones de dominancia y de dominados; v) el campo se visualiza así como un espacio de correlación de fuerzas históricamente determinado y cruzado por las estrategias de los agentes en función de mantener o cambiar tal correlación.

En una primera aproximación, pues, postular la edad como un campo implica, a lo menos, dos tareas centrales: i) analizar la edad en cuanto capital simbólico, ${ }^{4}$ y su desigual distribución entre agentes y posiciones dentro de este campo; ii) identificar los agentes principales que intervienen en el campo de la edad.

4 La edad es postulada aquí en cuanto campo social, pero, asimismo, constituye el capital simbólico en disputa dentro de este campo. Bourdieu distingue varios tipos de capital que son disputados en distintos campos: capital económico, social, cultural y simbólico. Este último, en una síntesis apretada, reúne los siguientes rasgos: i) se trata de un valor agregado, de una especie de propiedad suprasegmental que puede flotar adosada a las realidades de los otros capitales en la medida que es percibido en cuanto valor simbólicamente eficiente por agentes sociales que por lo mismo se lo disputan; ii) es un resultado de las relaciones de sentido existentes entre los individuos en una formación social dada y reúne en sí el conjunto de los reconocimientos socialmente validados en ese entorno; iii) por ello, dentro de los capitales, es el que se encuentra más desigualmente desplegado puesto que su desigual acumulación resulta más «invisible» que la del resto de los capitales. En el trabajo presente la edad es postulada en cuanto capital simbólico en disputa en el campo de la edad. 


\section{EL CAMPO DE LA EDAD}

\section{El subcampo de la longevidad}

\section{a) Cronos y la edad experienciada}

El sustrato de la edad reside en el reloj biológico de los individuos, en ese complejo sistema de tejidos, sistemas y subsistemas de orden químico, molecular y orgánico que se entreveran armónicamente, mientras no enferme y finalmente muera. La vida y su prolongación medida en años es el primer escalón de observación y estudio de la gerontología social. Envejecer es adentrarse en años, acumular años de existencia. Y eso es edad. En castellano los años son la propiedad de los individuos. Se tienen $\mathrm{x}$ años de edad. Los individuos poseen años. Desde el imaginario de la lengua castellana, no se flota ni se navega en el tiempo. Se detenta un tiempo personal que se mide en años de tener. No de ser. En este sentido la lengua inglesa, por ejemplo, se comporta de manera diferente. Frente a la edad, el idioma inglés afirma algo como "se es o está x años viejo (he's $\mathrm{x}$ years old)» o menos literal «se está x años envejecido (old)». El punto de referencia en este imaginario es el día del nacimiento: «desde el día en que se nace se está $\mathrm{x}$ años envejecido». El inglés refuerza esta idea cuando alude al cumpleaños usando el compuesto «birth/day», en castellano, literal, «día del nacimiento». «cumple/años», en cambio, es coherente con esa especie de exceso de «poseer» años. El concepto de cumplir - dentro del marco de esta lectura sincrónica de sugerencias lingüísticas en las que la lengua se hace cargo del fenómeno de la edad - refiere a la significación de «concurrir a hacer efectivo algo previamente comprometido». Hay aquí una especie de principio activo. Se viene a hacer efectiva la posesión de un año más. A cumplir con la ceremonia de tener un año más.

Independientemente del ethos que puedan sugerir diferentes sistemas lingüísticos frente al fenómeno de la edad biográfica, el hecho es que los sujetos viven y numeran sus existencias en unidades de habitar el mundo que el calendario ha puesto a su disposición. ${ }^{5}$ Con ello, en la

5 Así, teóricamente, un individuo nacido en Israel que cuenta sus años de existencia de acuerdo al calendario cristiano - calendario solar- a la larga va a contabilizar en su biografía — va a tener - más años que aquel individuo que en Israel registre sus años de existencia de manera estricta de acuerdo al calendario hebreo. Este último es un calendario 
sociedad moderna, la edad, uno de los tres elementos que conforman el núcleo más estrecho de la identidad personal - los otros son el sexo y el nombre - queda vinculado al sistema cultural de contar años del calendario en uso, una convención más, que discute así la ilusión lingüística de la edad como una posesión exclusivamente personal. Es más, muchos de los Estados modernos, en algún sentido, se apropian también del nacimiento de los individuos, y a cada recién nacido, se le asigna un número. La edad personal se transforma así en la edad para el Estado. El carné de identidad constituye el nacimiento del individuo para el Estado. Será el permiso de circulación del individuo en los laberintos del Estado y de la sociedad. De acuerdo a lo inscrito en el registro estatal, el individuo tendrá que en un estadio de su vida determinado, en un año calendario específico, estar en un cuartel o en una escuela. De no ser así estará en falta. El carné de identidad es el primer peldaño de la llamada edad social. Sobre esto se volverá más adelante.

Se toca aquí una primera distinción gerontológica que dice relación con el campo de la edad. Los individuos construyen sus existencias sobre la base de un desarrollo orgánico - que nos ubica en el mundo animal - y de la conciencia, que nos distingue al interior de ese mundo como una especie diferente a otras especies. Ambas realidades constituyen un continuo. La lógica del continuum biológico del desarrollo orgánico del cuerpo requiere en la actualidad marcos de referencia que hay que buscar en campos disciplinarios como la biología genética, las biotecnologías, la biomedicina. Las estrategias para aproximarse a esta realidad no son propiamente gerontológicas. El interés gerontológico en estas áreas del conocimiento tienen que ver con la pregunta qué tanto puede prolongarse la vida humana y de qué factores orgánicos depende su duración, cuestión que se vincula con los límites teóricos de la longevidad humana a la luz de los descubrimientos en curso sobre los principios que gobiernan la vida, medida en años.

Por otra parte y como es sabido, el continuo de la conciencia deja de ser tal, con la aparición y desarrollo en los individuos de la lengua. La aparición, ejercicio de la lengua - el habla - y su desarrollo permite nacer a la cultura, al mundo de las significaciones y de la interacción comunicativa. ${ }^{6}$

lunar y hay años que cuentan con doce meses y otros con trece. Estos últimos son llamados embolismales.

6 En el sentido que está contenido en el pensamiento del lingüista Ferdinand de Saussure y en las elaboraciones de Morris Berman. 
En este nivel, la edad es el núcleo de un campo de significaciones muy amplio integrado, entre otros, al dominio de la identidad personal de los individuos y de la dinámica de su percepción subjetiva.

\section{b) Cronos y la edad prometida}

La longevidad se presenta así como un subcampo del campo gerontológico de la edad y es aquel que se estatuye en función de la lucha social por la prolongación de la vida, es decir, por el control del envejecimiento y como consecuencia por el control de la prolongación de la vida. La longevidad es parte, entonces, de la legitimación de la edad como capital simbólico.

El control del envejecimiento de las personas y, por consiguiente, la producción de una mayor longevidad de los individuos, está vinculado, por una parte, a las estrategias de políticas públicas puestas en marcha y sostenidas, básicamente, por los Estados de Bienestar y, por otra, a las iniciativas de los agentes de las industrias farmacológicas, de las biotecnologías y de la ciencia médica.

Ambos desarrollos se superponen en el tiempo, crecientemente entretejidos por la modernidad, en la cual la vida cotidiana de los ciudadanos está cada vez más regida por la mezcla de prácticas estatales y de mercado y una tendencia poderosa hacia la globalización en la que los agentes privilegiados suelen ser entidades transnacionales de mercado y no precisamente los Estados.

Esta compleja situación ha insuflado entre los ciudadanos del primer mundo y de los que viven en economías nacionales emergentes la expectativa de una vida más larga.

No sólo la expectativa. En los Estados del Bienestar de los países del primer mundo se obtienen logros espectaculares en los últimos cincuenta años en la capacidad de crear y sostener condiciones de vida tales que permiten que los individuos alcancen en cantidades crecientes edades avanzadas. Así, un informe de Pfizer del año 2003 constataba que en España, la esperanza media de vida, alcanzaba a los 82,31 años, en Italia a 79,12 años y en Francia a 78,89 años. ${ }^{7}$ Asimismo, el Informe sobre la Salud año 2003 de la Organización Mundial de la Salud establecía que la esperanza media de vida al nacer en el mundo había pasado de los 46,5 años en 1950-55 a 65,2 en este siglo, valores que, sin embargo muestran una desigualdad irritante, dependiendo de

7 Recoletos Grupo de Comunicación: www.ondasalud.com. 
si se los mide en el seno de una sociedad desarrollada o al interior de una sociedad del tercer mundo. ${ }^{8}$ Acumular años, vivir más o vivir menos, es una cuestión de respirar o dejar de respirar, es cierto, pero, ya en este nivel, el reloj biológico muestra que dura más o que dura menos dependiendo del espacio social en el cual funciona.

Hoy, pues, nacer en el seno de una sociedad del primer mundo es un predictor, si no de inmortalidad, al menos de una vida articulada en la secuencia de muchos años, más años que nunca antes en la historia de la humanidad.

Pero hay más: la longevidad sin fin, la inmortalidad. Esto suena a sueños cercanos de algún tercer tipo, sin embargo, a caballo de la genética, de la ingeniería genética y de las biotecnologías, hay toda una industria que está embarcada en asuntos que rondan esta idea, por cierto, sin explicitarla.

A la vanguardia de este proceso se encuentra la investigación de la genética moderna y la experimentación con seres humanos, lo que implica, en principio, una osadía intolerable, al interior de patrones éticos. ${ }^{9}$

Las búsquedas de la genética y de las biotecnologías se introducen en los intersticios de los mecanismos que rigen el origen y la re-

8 El mismo Informe recién aludido ilustra esta desigualdad mediante los valores medios de esperanza de vida que muestran las mujeres japonesas: 85 años, frente a la esperanza media de vida de los hombres de Sierra Leona: 32 años.

9 «El avance vertiginoso de la Biotecnología y de la Informática, parece llevarnos de la mano a un nuevo mundo, donde la inmortalidad sería alcanzable. Vega, Vega y Martínez refieren que los embriones adquieren identidad humana solamente gracias a 'una adecuada interacción fisiológica con la madre', esto les ha llevado a sugerir que la diferenciación celular que da lugar a los tejidos del organismo adulto está dirigida por mensajes que provienen del organismo de la madre, de ser así, si en los incipientes embriones fueran inoculados con estas células capaces de auto-regenerarse, estaríamos, sin duda, a las puertas de la inmortalidad. Este proceso una vez iniciado, no tendría vuelta atrás: los seres humanos vendrían genéticamente programados para renovar sus órganos una y otra vez. Esta percepción del hombre como sujeto y objeto de experimentación en sí mismo, ante la ausencia de enfermedades y en el umbral de una longevidad jamás imaginada, conlleva una nueva concepción de la realidad con implicaciones políticas, sociales, económicas, religiosas y culturales. ¿Estaremos a las puertas del paraíso prometido o del desastre?». Luz Marina Pereira González «La inmortalidad: ¿un salto al vacío?», en www.galeon.com. 
producción de los tejidos vivos, para manipularlos. ${ }^{10}$ La exploración sistemática del patrimonio genético. Un eje en el que confluyen una serie de tecnologías. ¿Reprogramar a los seres vivos? De alguna manera. La neo-medicina o la medicina genómica, apoyada en el conocimiento acabado del genoma humano, refunda la manera de hacer medicina, es decir, la manera de tratar con las enfermedades, de cuidar y recuperar el cuerpo en una nueva ecología del envejecer.

La medicina no volverá a ser como antes. Su historia quedará partida en dos. La medicina pregenómica y la postgenómica. ${ }^{11}$

Con ello, en la lectura más determinista de esta revolución, la vida humana, la de cada individuo, se vuelve predecible. El futuro no

10 «Debemos esperar a la década de los 70 para que surja un conjunto de técnicas de laboratorio revolucionarias que por primera vez permiten 'tocar' de modo racional el sancta sanctorum de la vida. Son técnicas y herramientas con las que se puede modificar el ADN de acuerdo a diseños previos y objetivos concretos (de ahí el nombre popular de Ingeniería Genética). La Ingeniería Genética, mejor llamada tecnología del ADN recombinante in vitro, se caracteriza por su capacidad de cortar y empalmar genes o fragmentos de ADN de organismos distintos, creando nuevas combinaciones no existentes en la Naturaleza, combinaciones que ponemos a trabajar en el interior de una variedad de organismos hospederos, para nuestro provecho». Enrique Iáñez Pareja, «Introducción a la biotecnología», en: www.ugr.es.

11 «El desarrollo del Proyecto Genoma Humano (PGH) constituye una base importante de la medicina del futuro, la que algunos autores han denominado medicina genómica. El producto final del PGH será un mapa de referencia y secuencias de ADN que constituirá el libro de consulta para la biología humana y la medicina de los siglos venideros... Como decía el premio Nobel Watson (1990), 'nunca se encontrará un conjunto de libros de instrucción más importante. Cuando sean finalmente interpretados, los mensajes genéticos codificados dentro de nuestro ADN nos proporcionarán las últimas respuestas a los cimientos químicos de la existencia humana. No solamente nos ayudarán a comprender cómo funcionamos como seres sanos, sino que también nos explicarán, a nivel químico, el papel de los factores genéticos en una multitud de enfermedades - como el cáncer, la enfermedad de Alzheimer y la esquizofrenia - que disminuyen la vida individual de millones de personas'. Hasta ahora, la medicina ha sido preventiva, diagnóstica y terapéutica, pero con el advenimiento de la genómica, la medicina será también predictiva». Juan Ramón Lacadena, «El genoma: esperanza y amenaza», en: www.diariomedico.com. 
está ya más allá, en los astros, en las cosmogonías, en las líneas de la mano. No en un afuera. No en lo desconocido. El destino individual está aquí, en el presente de cada uno, impreso en los libros habitados por los genes. ${ }^{12}$

El factor predictivo de la medicina genómica constituye el trastorno más radical de la relación entre los seres humanos y preanuncia un cambio de era. Para los individuos, su plena instalación en el cotidiano próximo habrá de parecerse más a la situación del exilio de especie que a ninguna otra cosa. No habrá ya «pacientes», habrá, entre otros «in-pacientes». ${ }^{13}$ No es del caso ahondar en esto, por ahora.

12 «Cualquiera de nosotros puede estar sano en este momento, pero si nos hicieran un análisis de nuestro ADN podría descubrirse que tenemos una cierta probabilidad - siempre hay que transmitir la idea de predisposición o susceptibilidad, nunca de certezas genéticas deterministas- de cursar en el futuro un cáncer de colon, un cáncer de mama o la enfermedad de Alzheimer porque nuestro genoma es portador de determinadas mutaciones-》 (op. cit.).

13 «La introducción de la información genética sobre riesgos (probabilidad) de algunas enfermedades y la inevitabilidad (certeza) de otras, así como la severidad cambiante de estas condiciones y la multiplicidad de factores que influyen en su expresión, puede alterar los términos de la relación médico-paciente en dos aspectos diferentes que Jonsen denomina «el paciente como población» $\mathrm{y}$ «el problema del in-paciente».

a) El paciente como población. Se refiere a que la familia del paciente pueda pasar de ser una comunidad de apoyo al enfermo a ser una comunidad de personas afectadas: las familias serán consideradas como focos de enfermedad, como portadores de riesgo conocido o de enfermedad inevitable. Ello llevará consigo que las decisiones reproductivas (tener o no tener descendencia) llegarán a ser tan significativas en el pensamiento terapéutico como lo son ahora la utilización de fármacos o la cirugía. El paciente será visto quizás menos como individuo que como «unidad de población».

b) El problema del in-paciente. Se refiere a aquellos casos en los que la información genética obtenida del análisis molecular permitirá conocer a la persona afectada su futuro mucho antes de que se presenten los síntomas de una enfermedad para la cual no existe en el momento actual una terapia eficaz. Es decir, hay que distinguir entre los no-pacientes -que no entran en el mundo clínico porque están sanos-y los in-pacientes que, pudiendo entrar en el mundo clínico, no pueden ser curados». Juan Ramón Lacadena, El proyecto genoma humano: Consideraciones éticas, en: www.cnice.mecd.es. 
No obstante este contexto de avances impactantes en el conocimiento de la realidad orgánica, el envejecimiento de los organismos vivos no es todavía explicable. ${ }^{14}$ Sobre ello, la biogerontología reconoce una multiplicidad de teorías causales del envejecimiento, ninguna de las cuales, sin embargo, muestra adelantos definitivos al respecto.

Las investigaciones en curso en la genética, la medicina genética, las biotecnologías y la nanociencia o nanotecnologías, a lo menos instalan la pregunta sobre qué tan longeva podría llegar a ser la vida humana sin las enfermedades que conforman hoy los perfiles de mortalidad más típicos.

\section{El subcampo de las clases de edad}

Si la lucha por la prolongación de la vida convoca a agentes muy diversos al interior de un espacio específico dentro del campo de la edad, las prácticas por categorizar y valorar las edades así como las prácticas de reproducción social que se da entre ellas apuntan a deslindar un segundo espacio dentro del cual se producen las edades. La instalación de las clases de edad en un universo social dado es el resultado de un proceso histórico particular. Por ejemplo, en las sociedades de occidente, la infancia recién es percibida socialmente en cuanto clase de edad a fines del siglo XVII. ${ }^{15}$ Hasta ese entonces, los

14 Existen varias teorías sobre las causas del envejecimiento celular sin que sea posible adherir a un solo marco explicativo. Las teorías existentes se clasifican en dos grandes grupos: a) el envejecimiento es producto de alteraciones acumulativas a lo largo del tiempo: teorías estocásticas. En este grupo se sitúan las construcciones conocidas como: a.1) teoría del error catastrófico; a.2) teoría del entrecruzamiento; a.3) teoría del desgaste; a.4) teoría de los radicales libres; b) el envejecimiento es genético: teorías no estocásticas. Dentro de esta línea argumentativa se encuentran las siguientes teorías: b.1) teoría del marcapasos; b.2) teoría genética. (Hoyl, 2002).

15 «A fines del siglo XVII, cambia el sentido de la niñez: los niños llegan a conformar un mundo aparte del de los adultos, y se transforman en actores sociales. Tres grandes acontecimientos hacen posibles tales cambios: i) La disminución de la mortalidad infantil, debido a la preocupación por la higiene del cuerpo, y por los alimentos. Durante la Edad Media y bien avanzada ésta, por falta de higiene, de cada 100 niños morían 60 ó 70 en el primero, o en los dos primeros años de vida. También morían muchas madres por las fiebres puerperales, infección que duró 
niños vivían revueltos con los adultos. ${ }^{16} \mathrm{~A}$ su vez, la clase de edad «adulto mayor» recién emerge en el siglo pasado.

Las clases de edad se instalan, pues, como el segundo eje de cristalización del capital simbólico edad al interior del campo con el mismo nombre.

Al respecto, y siguiendo en ello a Enrique Martín Criado, las clases de edad son construcciones sociales vinculadas al concepto de edad social, definible como «serie de derechos, privilegios, deberes, formas de actuar [...] —en suma, por una 'esencia social' - y delimitada por una serie de momentos de transición - que difieren históricamente: matrimonio, servicio militar, primera comunión, certificados de escolaridad-. A su vez, cada grupo social establece una serie de normas de acceso - más o menos codificadas y ritualizadas en forma de 'ritos de paso' - de una clase de edad a otra» (Martín Criado).

Como se ve, Martín Criado rescata para estas categorías una cierta «esencia social» oponiéndose explícitamente a conferirles alguna «esencia sicológica»: «no dependen de una serie de 'naturalezas psicológicas' previas, sino que se construyen en el seno de cada grupo social en función de sus condiciones materiales y sociales y, sobre todo, de sus condiciones y estrategias de reproducción social» (op. cit).

Vale decir, el concepto de edad social complejiza, tensiona, desestructura y desafía tanto al sentido común como a los imaginarios que rondan y han rondado la categoría edad, proponiéndola como una construcción social y cultural a través de la cual al interior de las sociedades los diferentes grupos sociales organizan sus actividades.

Para el imaginario de la antigüedad clásica, por ejemplo, el ciclo de vida de los seres humanos tenía tres edades. La mítica Esfinge las sintetizaba sosteniendo que el hombre era aquel animal que caminaba al amanecer en cuatro patas, en dos hacia el medio día y en tres hacia el anochecer. Infancia, adultez y vejez, tres etapas del desarrollo humano, tres etapas del volverse viejo y una sola manera de vivir la

mucho tiempo. ii) La aparición de la imprenta. iii) La aparición de la escuela básica a la que los niños iban a aprender los números y las letras» (Barbero, 2002:170).

16 «Según el famoso historiador francés Philippe Ariès, quien ha hecho la historia de la infancia en Occidente, hasta el siglo XVII, durante toda la Edad Media, y bien avanzado el Renacimiento, los niños vivieron revueltos con los adultos: revueltos en el trabajo, revueltos en la iglesia, revueltos, incluso, en la cama» (Íbid). 
vejez: en tres patas. Para la voraz Esfinge, vejez y ortopedia, una misma fase.

Un conjunto de factores ligados en especial a la cultura moderna han determinado que las fases del ciclo vital tiendan a ser percibidas de una manera más diversificada y sustancialista. Habría una manera de existir en el espacio que definiría «lo joven», «lo infantil», «lo viejo». Distinciones tales como infancia subdividida en primera infancia e infancia, adolescencia, preadolescencia, juventud, adultez, adulto joven, adulto mayor, etc., legitiman la fantasía de la naturalización de etapas universales del ciclo vital.

Este último fenómeno es el resultado de la actuación de agentes en el campo de la edad que apuntan en esa dirección de manera simultánea a lo menos en dos órdenes de cosas: el mercado y el del desarrollo de las ciencias humanas, en especial del área de la psicología evolutiva.

En el primer orden de cosas cabe aludir la mercadotecnia que ha impuesto microculturas vinculadas a las etapas del ciclo vital vigorosas. La adolescencia es hoy, por ejemplo, un punto de referencia importante de la publicidad y desde allí, pero también desde otros puntos estratégicos de la industria del consumo, se han gavillado verdaderos enclaves, auténticos imaginarios, para vivir como «adolescente»: música, modas vestimentarias, alimentarias, de intervenciones corporales, prácticas sociales, rituales de encuentro, espacios públicos.

Este alcance a los agentes de la mercadotecnia, todos ubicados en el polo del incentivo del consumo es a su vez la cristalización de procesos históricos complejos desarrollados en los siglos XIX y XX, en especial, con la constitución y consolidación democrática o masiva de instituciones como la escuela y la universidad. En esos espacios sociales, niños y jóvenes, han aprendido y han madurado estrategias y prácticas para modificar las pautas de control de las generaciones adultas sobre las más jóvenes, cambiándolas en función de una mayor autonomía y de construcción de identidades vigorosas en la afirmación de protagonismos que les habían sido negados.

Las prácticas sociales de niños y jóvenes en las sociedades del primer mundo han transformado en los últimos cincuenta años de manera sustancial los contenidos de las edades sociales a ellos adscritos por un mundo adulto tendencial y crecientemente absorbido por la necesidad de la productividad.

Por otra parte y en el mismo período, las ciencias sociales identifican y desarrollan poderosamente las identidades diferenciadas de la 
niñez y de la adolescencia como fases diferenciadas del desarrollo humano, independizándolas del punto de referencia de la adultez al cual habían permanecido ligadas por siglos.

Los hallazgos realizados a nivel de las ciencias vinculadas a la educación, y en especial las construcciones de la psicología evolutiva, describieron e instituyeron fases o edades del ciclo vital mucho más matizadas que las del imaginario de la devoradora de hombres, la Esfinge.

Ontogenia - observación de la evolución de los seres vivos desde su nacimiento hasta su muerte- y observación del decurso del proceso de individualización de los sujetos de la especie humana, psicología evolutiva, han puesto en escena el ciclo vital. No obstante, sus etapas difieren dependiendo del teórico que las describa. Wallon establece seis estadios de desarrollo, desde el nacimiento ( 0 año) hasta la adolescencia (que comienza con los doce años) para lo que ontogénicamente se enuncia como períodos de desarrollo y madurez. Piaget distingue cuatro fases para los mismos períodos. Erikson, en tanto, fundamenta ocho etapas. ${ }^{17}$

La puesta en escena de las edades en las sociedades modernas, sin embargo, va de la mano de la irrupción del protagonismo juvenil en el ámbito de las decisiones políticas, en las discusiones sobre el destino de los espacios sociales e históricos habitados por los más jóvenes, en las visiones que son capaces de crear, opuestas o distintas a las propuestas de los mayores. Es el protagonismo juvenil instalado

17 Wallon: i) Estadio impulsivo (desde el nacimiento hasta los cinco o seis meses); ii) Estadio emocional (desde los seis meses hasta completar el año); iii) Estadio sensorio motriz y proyectivo (1 a 3 años); iv) Estadio del personalismo (3 a 6 años); v) Estadio categorial (6 a 11 años); vi) Estadio de la adolescencia. Por su parte, Piaget establece las siguientes fases en el desarrollo que está referido básicamente a la infancia/adolescencia: i) Período sensomotriz (desde el nacimiento hasta aproximadamente los dos años); ii) Período del pensamiento preoperacional (2 a 7 años de edad); iii) Período de operaciones concretas (7 a 11 años); iv) Período de operaciones formales (11 a 15 años). Por último, las ocho etapas que distingue Erikson son: i) Etapa del bebé (primer año de vida); ii) Apertura a la relación con ambos padres (segundo año de vida); iii) Apertura a las relaciones con la familia básica ( 3 a 5 años de edad); iv) Apertura a las relaciones de la escuela y con vecinos (6 a 12 años de edad); v) Adolescencia; vi) Primera juventud; vii) Juventud y primera madurez; viii) Adultez. 
en las prácticas sociales, entonces, lo que pone en cuestión de manera directa, una concepción de la edad que los mayores manejan en función del control social de los menores (Feixa, op. cit.). En otras palabras, lo que pone en crisis la emergencia del sujeto social juvenil es la construcción de la clase de edad en la cual los jóvenes tendrían que caber, es el conjunto de deberes, ritos y prácticas dables de esperar de ellos, dada la clase de edad a la cual se encuentran adscritos, construida entre otras consideraciones, por las sugerencias provenientes de las periodizaciones etarias del ciclo vital. ${ }^{18}$

En otras palabras, desde la perspectiva del campo de la edad, el capital simbólico de la edad reconoce en la lucha por definir los contenidos y estatutos de las clases de edad uno de los enclaves centrales desde el cual, la edad, en cuanto capital simbólico, es definido y administrado.

Infancia, juventud, adultez, vejez no refieren a ningún contenido predefinido per se y devienen en categorías sociales y culturales, es decir, en capital simbólico de la edad, al interior de las luchas que los distintos agentes del campo establecen en función de mantener o cambiar los contenidos que definen las clases de edad.

Una gerontología social, preocupada de dar cuenta del estado del campo de la edad en un momento dado, de acuerdo a la perspectiva que sigue nuestro planteamiento, habrá de caracterizar cada una de las clases de edad en función de las relaciones que guarden entre ellas y en función de su carácter de clase de edad dominante o subordinada, además de dar cuenta de los factores que permiten la reproducción de ese estado y/o de los elementos que juegan para su reemplazo. ${ }^{19}$

En esta tarea, la gerontología social habrá de preguntarse por la posición que ocupa al interior del campo de la edad, el capital simbó-

18 Así, por ejemplo, desde la normativa social que enmarca y alimenta los contenidos de la clase de edad «juventud» de los grupos medios y altos de las sociedades latinoamericanas, la pregunta de un joven que ha terminado la secundaria: «¿trabajaré o estudiaré?» es socialmente pertinente. La pregunta no lo sería en boca de un/una envejeciente adscrito/a a la clase de edad «adulta», porque la práctica de «estudiar», no está asociada normativamente a esa clase de edad.

19 La categoría de edad social aquí es tratada como un concepto relacional. Mayores y más jóvenes, son términos distinguidos en la práctica social de acuerdo al régimen de prohibiciones/otorgaciones explícita o implícitamente instalado de acuerdo a las especificaciones históricas que asuma la estructura de dependencia/autonomía/exclusión/inclusión que enmarca las relaciones entre clases de edad. 
lico de la vejez. Ello en función de los dos subcampos esbozados: el de la longevidad y el de las clases de edad.

En términos generales, y es sólo una hipótesis, el capital simbólico de la vejez, al interior del primero de los subcampos, aparece subsumido y desdibujado en el orden del deseo: vivir una larga vida. Las apuestas de los agentes en el subcampo de la longevidad constituyen un ponerse con la «vida» y en las que la condición de realidad de las mismas está constituida por la interdicción de la vejez, por erradicar del campo de la edad a la vejez, lo que es lo mismo que poner como condición la negación de la vejez como integrante del valor simbólico de la edad.

En el subcampo de la longevidad, la vejez está ubicada claramente en el paradigma dominado del campo de la edad, puesto que las estrategias de los agentes en sus apuestas coinciden desde diversas posiciones a valorizar el capital simbólico de la edad más cercano a la frontera de la adultez/juventud como aquel capital por el cual hay que luchar.

En el subcampo que se describe, el capital simbólico de la edad por el cual se lucha está asociado, en sus modulaciones más moderadas, a la postergación más extrema de la aparición de la enfermedad, a la perduración de la sexualidad, de la capacidad de aprender, de conservación de las funcionalidades vitales, y en sus modulaciones más extremas, a la erradicación radical de las enfermedades, a la conservación ilimitada de las funcionalidades, etc.

Como se ve, desde las manipulaciones posibles de producción de las edades en el subcampo de la longevidad, la tendencia reside, en su versión más moderada a morir después de una larga vida como adulto/joven (nunca más a morirse de viejo). En su versión más extrema, en el extremo de la edad prometida, a no morir.

Por otra parte, desde la perspectiva del subcampo de las clases de edades, la vejez, en la mirada contemporánea, constituye un componente del capital simbólico de la edad inscrito en el paradigma de la subordinación.

Su ubicación al interior de este paradigma no deja, sin embargo, de causar inquietud. Por varias razones: los longevos poblarán la tierra en una mayor proporción que los menos longevos; su proliferación pondrá en duda la capacidad de los menos longevos de sostener el aparato productivo sin la colaboración de los más longevos; las actuales tendencias de los más longevos, inducidos por el funcionamiento actual de las sociedades modernas, a refugiarse en los espacios del 
retiro funcional social, ponen en duda la capacidad de sustentabilidad de las sociedades modernas.

Más allá de esta colección de miedos, el capital simbólico etario de los más envejecidos, en las actuales circunstancias, está puesto en duda al interior de la estructura de las edades sociales de las sociedades modernas, en la medida que su valor como capital simbólico de la edad, se encuentra limitado en su relación con las otras edades de manera drástica. Así, podemos observar que: i) Los criterios del desgaste, del cansancio vital y del deterioro orgánico, que fundamentaron en sus orígenes la necesidad del retiro de los más envejecidos. A finales del siglo XIX y bajo el reinado de un capitalismo bárbaro, la posibilidad de abandonar el trabajo y percibir un salario, fue una auténtica conquista. ${ }^{20}$ ii) Por otra parte, en una sociedad crecientemente industrial y urbana, el derecho adquirido tenía una contraparte vinculada al dinamismo de la lucha entre las clases de edades: quien deja un empleo - el más envejecido - permite que otro - menos envejecido- obtenga un empleo. El derecho de los más envejecidos puede mirarse también como la astucia de una estrategia de la clase de edad de los adultos para desplazar del mercado del trabajo a los adultos más envejecidos. iii) El capital simbólico de la edad se encuentra así asimétricamente dispuesto al interior de un campo — la edad - en el que el espacio de la dominación es ejercido por todos aquellos adscritos a la clase de edad de los adultos. ${ }^{21}$

\section{El subcampo de las generaciones}

Frente a las clases de edad, las generaciones constituyen grupos de individuos situados en el espacio social de una manera diferente. Mientras las primeras están visibles y cristalizadas en el régimen de prohibiciones sociales/licencias sociales validado, las generaciones remiten «a las variaciones estructurales en el tiempo, dentro de un

20 Se alude aquí a los inicios del concepto de seguridad social y de las referencias básicas del estado bienestar. Así, en 1883 se implantaron los primeros programas estatales de previsión social en la Alemania de Bismarck. Dicha legislación estableció las bases de la moderna seguridad social bajo el principio contributivo, mediante el cual se financiaba obligatoriamente un sistema básico de previsión social.

21 En esta dirección, varios autores (Feixa, Martín Criado, entre otros) enmarcan esta idea bajo el concepto de adultocentrismo. 
campo, de los modos de generación de sujetos». Por ello, «la extensión en el espacio social e histórico de una generación dependerá de las categorías del observador y de la extensión o restricción de lo que se entienda como 'homogeneidad de las condiciones de existencia'» (Martín Criado, op. cit.).

El interés gerontológico en las generaciones proviene básicamente de dos de las necesidades que fundan a la gerontología social en cuanto disciplina social y no sólo como un campo de preocupaciones interdisciplinario, a saber: i) la necesidad de explicar los mecanismos y procesos que puedan dar cuenta de la reproducción o del cambio de los contenidos de las clases de edad en un momento específico de estructuración del campo de edad; ii) la necesidad de dar cuenta de la manera de producción específica de los individuos más envejecidos en el campo de la edad. Todo ello en la medida que se entienda por generación:

a) Un grupo de sujetos cuya identidad no está dada por la contemporaneidad cronológica - coincidencia en los años de nacimiento- que vivencian sino, más bien, en la posibilidad que tal circunstancia abre de ser partícipes de escenarios vitales similares. ${ }^{22}$ En esta definición, el compartir «un mismo cuadro de vida histórico social» constituye un rasgo fundante del concepto «generación» situado al mismo nivel jerárquico que el de la coincidencia de nacimiento.

No obstante, ello complejiza la instrumentalización del concepto en la medida en que, en la tradición de las ciencias sociales, este concepto ha oscilado en su aplicación a realidades muy diferentes según el contexto disciplinario en el cual se use y las restricciones de tiempo y espacio social que detente el grupo al cual se implique: «generación del 98» (historia de la literatura/grupo de escritores en lengua española cuya escritura tiene como motivo común de preocupación la situación de la España de fines del siglo XIX y nacidos entre 1864 y 1875; «generación del 79» (historiografía/clases de edad en Chile que vivieron la guerra entre chilenos, peruanos y bolivianos). ${ }^{23}$

22 En palabras de Mannheim, coincidir en el año del nacimiento constituye «la posibilidad que se sigue de participar en los mismos acontecimientos, en la misma vida, etc. y, más aún, de hacerlo a partir de una misma forma de estratificación de la consciencia... Sólo un mismo cuadro de vida histórico-social permite que la situación definida por el nacimiento en el tiempo cronológico se convierta en una situación sociológicamente pertinente» (Mannheim, 1990:52-53).

23 «La extensión de una generación en el espacio social puede variar de un grupo restringido de pretendientes en tal o cual campo (vanguardias li- 
De aquí, a lo menos, dos consecuencias metodológicas de pertinencia gerontológica.

i) Lo dicho, en la concepción de Bourdieu, que inspira la presente propuesta, implica que una generación, al interior del campo de la edad, refiere a un conjunto de sujetos, en principio, heterogéneos que les ha tocado compartir un mismo tiempo histórico, el que han procesado, sin embargo, de manera diferente, dependiendo de las variables de la posición que ocupan dentro del campo de la edad, — habrá individuos que teniendo sesenta años, por ejemplo, están estableciendo una pareja nueva, adquiriendo compromisos de paternidad, mientras que otro conjunto de individuos de la misma edad, podrán haber finalizado sus tareas de maternidad o de paternidad - del espacio social, - porque unos pertenecen a la clase dominada y otros a la dominante- etc. De aquí que una misma edad, como ha sido dicho, no sea garantía de la constitución de generaciones específicas ni de la producción de sujetos ni de agentes semejantes.

ii) Las generaciones pueden constituir una construcción metodológica de la gerontología social privilegiada porque tal construcción le posibilita al observador resituar la variable edad - la contemporaneidad - al interior de un haz complejo de variables - posición, clase, sexo- que son precisamente las que constituyen el campo de la edad, dando origen, de esa manera, a una construcción mucho más apta que otras del campo de la edad, ${ }^{24}$ para dar cuenta de la idea que sustenta la

terarias o artísticas) a la cuasi-totalidad de una clase de edad (como, en caso de guerra, en la movilización de los soldados de un contingente) [...] de la entrada en el mismo momento en una misma profesión (que supone un mismo 'modo de generación') a la simple participación en un mismo 'acontecimiento-fundador' (como una guerra o una crisis política: la guerra de Argelia o Mayo 68), de la confrontación a una misma situación (la crisis del mercado de empleo, por ejemplo) de toda una clase de edad, a la situación específica de los titulares del mismo sexo de tal categoría de diploma» (Mauger, 1990:11; citado por Martín Criado, op. cit.).

24 Como ya se ha perfilado, los usos del concepto de generación en las ciencias sociales es profuso, no obstante incómodo para una gerontología social que habla de la producción de las edades. En ocasiones equivale al concepto de clase de edad, en otras al de cohorte, en otras a una mayoría estadística de comportamientos análogos al interior de un rango de edades, en otras a un grupo etario que comparte explícitamente sensibilidades o ideologías. 
disciplina gerontológica, a saber, el fenómeno de la producción de las edades.

b) Contemporaneidad, la extensión acotada de una generación en el espacio social, ${ }^{25}$ y posición en el campo de la edad aluden a los componentes de cómo se está entendiendo el concepto de generación. Habría que agregar a ello, el rasgo de un grupo de sujetos que posee una «situación análoga... en el espacio social» más que un grupo concreto poseedor de un lazo social entre sus miembros que los liga e identifica, aunque ello no sea descartable (Mannheim, 1990:41).

\section{Habitus, clases de edad y generaciones}

Junto a los rasgos aludidos, otro de los requisitos fundadores del grupo generacional - ya mencionado más arriba sin que se le haya relevado suficientemente - se vincula con la construcción de las subjetividades por parte de los sujetos que componen una misma generación. «Participar en unos mismos acontecimientos» + «a partir de una misma forma de estratificación de la consciencia», decía Mannheim (ver nota 24$)$.

Este último elemento, la «misma forma de estratificación de la consciencia» puede asimilarse en la presente argumentación al segundo elemento central de la teorización de Bourdieu que se viene siguiendo: el habitus, ya mencionado más arriba.

Como también ha sido ya citado, a nivel gnoseológico, la aproximación a la realidad de Bourdieu es, básicamente, hereditaria del estructuralismo. La realidad se articula en relaciones. «Si todo lo real es

25 Dado lo extendida que se encuentra una concepción de las generaciones como conjunto que representa toda la extensión del espacio social en un momento determinado se hace necesario insistir en la inexactitud de la misma, aun cuando se trate de «una serie de acontecimientos fundadores «una guerra, una crisis económica, la extensión del sistema escolar, etc.» que afectaría por igual a todo el espacio social [...] Porque estos acontecimientos - aun los más brutales $\mathrm{y}$, en principio, más globalizantes, como las guerras - tienen efectos muy distintos según la estructura del campo y la posición en el espacio social en que se hallen los sujetos. En vez de partir de totalizaciones — «la generación del 68», etc.- buenas para los relatos periodísticos, pero nefastas para los sociológicos, lo que hay que plantearse es los efectos, en cada campo particular, y para cada grupo de agentes, de un acontecimiento que en principio abarca a todo el espacio social» (Martín Criado, op.cit.). 
relacional [...] es necesario pensar relacionalmente» (Bourdieu y Wacquant 1995). De esta manera, habitus y campo son elementos que se suponen mutuamente al interior de la teoría de Bourdieu, constituyendo las matrices centrales de toda su construcción. De otra manera, no hay campo sin habitus ni hay habitus sin campo.

¿Cómo orientan sus actuaciones al interior de cada campo los agentes que allí operan?

Se hace necesario en esta parte de la argumentación ampliar los conceptos básicos de la construcción bourdesiana brevemente anotada con anterioridad. Como se recordará, según Bourdieu, cada sujeto o agente orienta sus actuaciones al interior de un campo dado, a partir de sus respectivos habitus definidos en cuanto sistemas «de disposiciones durables y transferibles - estructuras estructuradas predispuestas a funcionar como estructuras estructurantes - que integran todas las experiencias pasadas y funciona en todo momento como matriz estructurante de las percepciones, las apreciaciones y las acciones de los agentes de cara a una coyuntura o acontecimiento y que él contribuye a producir» (Bourdieu, 1991). Tales esquemas se caracterizan por:

a) Funcionar «más allá de la conciencia y del discurso». En este sentido, el habitus refiere a «principios generadores y organizadores de prácticas y representaciones que pueden estar objetivamente adaptadas a su fin sin suponer la búsqueda consciente de fines y el dominio expreso de las operaciones necesarias para conseguirlos, objetivamente 'reguladas' y 'regulares' sin ser para nada el producto de la obediencia a reglas, y siendo todo esto, objetivamente orquestadas sin ser el producto de la acción organizadora de un jefe de orquesta» (Ibídem). Su función elemental consiste en orientar «prácticamente las prácticas».

b) Formar parte del organismo, del cuerpo, de los sujetos. El habitus está literalmente in/corporado a ellos. El habitus se hace corporeidad producto de su exposición a condicionamientos de muy variada naturaleza, formales e informales, pero en especial, aquellos de una pedagogía invisible («implícita»). ${ }^{26}$

26 Una pedagogía «capaz de inculcar toda una cosmología, una ética, una metafísica, una política, a través de órdenes tan insignificantes como 'ponte derecho' o 'no cojas tu cuchillo con la mano izquierda' y de inscribir en los detalles en apariencia más insignificantes del porte, de la postura o de los modales corporales y verbales los principios fundamen- 
c) Ofrecer a los sujetos las nociones más elementales de organización de lo social. ${ }^{27}$ tancia. ${ }^{28}$

d) En su constitución, por cierto, la infancia tiene capital impor-

e) En relación al campo, el habitus supone la existencia en los sujetos y en los agentes del interés por hacer sus apuestas en el campo específico. El interés no está de manera natural en los agentes sociales ni tampoco es el resultado de la reflexión. Existe en la medida en que ha sido incorporado por éstos a través de la práctica, vía por la cual también se han apropiado de los esquemas regulatorios de apuestas y funcionamiento del campo. ${ }^{29}$

Para la observación del campo de la edad, así como, por cierto, en la construcción teórica de Bourdieu, el concepto de habitus posee un carácter estratégico. Básicamente permite cerrar la comprensión de cómo se articulan en su interior las relaciones entre «condiciones materiales, capital simbólico, posiciones existentes en el campo»y «comportamientos de los agentes» en el proceso de producción de las edades. Las condiciones estructurales existentes en el campo no son adosables mecánicamente a las voluntades, deseos, elecciones, necesidades, de los sujetos que operan en cuanto individuos en el campo.

tales del arbitrario cultural, situados así fuera del alcance de la consciencia y de la explicitación» (Ibídem).

27 El habitus contiene «los principios más fundamentales de la construcción y de la evaluación del mundo social, aquellos que expresan de la forma más directa la división del trabajo entre las clases, las clases de edad y los sexos o la división del trabajo de dominación» Esto por cuanto el habitus se origina en «condicionamientos asociados a una clase particular de condiciones de existencia» (Ibídem).

28 En la infancia «en primer lugar la casa es el lugar privilegiado de la objetivación de los esquemas generadores y, por medio de las divisiones y de las jerarquías que establece entre las cosas, entre las personas y entre las prácticas, este sistema de clasificación hecho cosa inculca y refuerza continuamente los principios de la clasificación constitutiva del arbitrario cultural» (Ibídem).

29 En palabras de Bourdieu «mediante la inmersión en un universo de prácticas que define lo que está en juego, lo que vale la pena: en otras palabras, porque han incorporado en su habitus - y por tanto, más allá de su reflexión y conciencia - unos esquemas apreciativos y evaluativos particulares» (Bourdieu, 1990). 
Sus prácticas están mediadas por sus habitus, por patrones conductuales modelados en su origen por las condiciones sociales y materiales en las que los sujetos sociales existieron así como por la clase social, la posición y el género desde las cuales fueron vivenciadas.

De esta manera, el habitus media las prácticas que niños, adultos y mayores evidencian al interior del subcampo de las clases de edad, así como las estrategias que cada uno de los agentes sociales de las clases de edad pone en juego para conservar o mejorar sus posiciones relativas en función del capital simbólico que manejan.

En este sentido, al interior del subcampo de las clases de edad, es dable postular la existencia de habitus específicos in/corporados en los sujetos que integran cada una de las clases de edad cuyos contenidos pueden y deben ser explorados por una Ciencia del Envejecimiento. Dentro de ella, a la Gerontología Social, entre otras operaciones, le correspondería estudiar y describir el habitus de los envejecientes más envejecidos.

No obstante, dado que la pregunta por el habitus de la post adultez no puede ser explorada ni contestada sino al interior del haz de relaciones que la post adultez guarda con el resto de las clases de edad al interior del subcampo de la edades, la pregunta por el habitus de la post adultez instala simultáneamente una pregunta por la estructuración del subcampo y más allá de él por el campo de la edad.

De aquí es también cierto afirmar que en un estado dado del subcampo de las clases de edad, cada distinción practicada en este subcampo - lo joven/lo adolescente/lo niño/lo viejo - habrá de instalarse en el subcampo de las clases de edad en función de habitus diferenciados.

La explicitación del concepto de habitus tiene consecuencias también para la tarea y las funciones que como agentes sociales al interior del campo de la edad cumplen las generaciones, es decir, tiene efectos para el análisis del subcampo de las generaciones. A diferencia de las clases de edad, el habitus generacional, es el resultado de la producción de generaciones nuevas producto a su vez del cambio de las condiciones materiales y sociales de la producción de los sujetos: «la transformación del modo de generación social de los agentes determina la aparición de generaciones diferentes» (Bourdieu, 1988). Sin condiciones sociales y materiales nuevas no habría propiamente generaciones sino sólo clases de edad. Como lo explica Martín Criado (op. cit.), «en una sociedad completamente estática, en la que las condiciones sociales y materiales con que se encuentran los nuevos miembros son idénticas a las que se encontraron los viejos en su tiem- 
po, no habría diferencias de generación: las diferencias entre 'jóvenes' y 'viejos' serían meras diferencias de clases de edad».

El habitus de cada generación, pues, se corresponde con cambios ocurridos en el espacio social en las condiciones de existencia. ${ }^{30}$

Una gerontología social debiera investigar los habitus generacionales para explicarse desde allí los cambios que pudieran evidenciarse en las definiciones de las clases de edad al interior del campo de la edad, poniendo el énfasis en la clase de edad de los más envejecidos.

$\mathrm{Si}$ es que las clases de edad y los habitus respectivos de quienes las componen se corresponden con ciertas condiciones de existencia, el estado del campo de la edad habrá de modificarse por la emergencia de habitus nuevos introducidos en el campo de la edad por la producción de nuevos contingentes de sujetos al interior de condiciones de existencia diferentes. La gerontología social habrá de preocuparse de manera preferente de construir su objeto de estudio en torno a las generaciones y sus habitus respectivos dedicando especial énfasis a tomar nota de las condiciones sociales, económicas, políticas y culturales de producción de los más envejecidos.

La rigurosidad metodológica que ponga en este afán habrá de permitir la emergencia de una disciplina social capaz de generar sus propias herramientas analíticas, demarcar fronteras, en especial con las disciplinas más afines, y tender a consolidar su pertinencia.

En el intertanto, las modulaciones teóricas de Bourdieu, sirven de punto de arranque.

\section{LOS AGENTES DEL CAMPO DE LA EDAD}

Hasta ahora, el campo de la edad ha sido postulado como estructurado en tres subcampos: a) el subcampo de la longevidad; b) el subcampo de las clases de edad; c) el subcampo de las generaciones.

El análisis gerontológico debiera orientarse a especificar, en cada subcampo, el tipo de capital simbólico de la edad que está en juego - el de la longevidad, clases de edad, generacional-, el establecimiento de los paradigmas de la dominancia y de lo dominado, y las estrategias que en cada subcampo ponen en movimiento los agentes que actúan en el subcampo en función de la conservación o del cambio de las posiciones en juego.

30 Como se sabe, todo habitus se origina en «condicionamientos asociados a una clase particular de condiciones de existencia» (Bourdieu, 1990). 
Para cumplir con estas referencias metodológicas que debieran dar cuenta de la producción de las edades, del envejecimiento y del estatuto de los más envejecidos, falta identificar los agentes ${ }^{31}$ que actúan en los subcampos, describir sus habitus y estrategias y por cierto, sus coordenadas posicionales (ver Cuadro 1 «Agentes y subagentes en el campo de la edad).

Cada campo instalado en el espacio social de una sociedad dada es el producto de luchas específicas por capitales específicos entabladas por agentes que disputan por la conservación de las estructuraciones existentes en el campo o por subvertirlas.

¿Quiénes son los agentes que de manera indirecta o directa influyen, «juegan sus apuestas» en el campo de la edad?

A continuación y sin mayor análisis ni elaboración se identifican algunos de esos agentes en función de los subcampos en los cuales operan.

Queda pendiente la tarea de especificar el estatuto de estos agentes y subagentes así como describir sus estrategias de operación a fin de terminar de visualizar el campo de pertinencia de la gerontología social.

SANTIAGO (CHILE), MAYO 2006

RECIBIDO: MAYO 2006

ACEPTADO: JULIO 2006

\section{REFERENCIAS BIBLIOGRÁFICAS}

BARBERO, JESÚS MARTíN (2002): «Comunicación y construcción social de las edades.» En: VV.AA.: Periodismo y comunicación para todas las edades. Bogotá: CEPSIGER.

BERMAn, MorRIS (2004): El reencantamiento del mundo. Santiago: Cuatrovientos.

BOURDIEU, PIERRE (1991): El sentido práctico. Madrid: Taurus. (1990): Sociología y cultura. México: Grijalbo.

_ (1988): La distinción. Crítica social del gusto. Madrid: Taurus.

31 Bourdieu se refiere al agente como aquel —agente social o institución-que «sufre y produce efectos en él mismo» (Bourdieu y Wacquant, 1995:173). 
(1969): «Campo intelectual y proyecto creador». En: VV.AA.: Problemas del estructuralismo. México: Siglo XXI.

— y LOÏC WACQUANT (1995): Respuestas, por una antropología reflexiva. México: Grijalbo.

De SAussure, Ferdinand (1983): Curso de lingüistica general. Madrid: Alianza Editorial.

— Feixa, Carles: «Antropología de las edades». En: Biblioteca Virtual de Ciencias Sociales, www.cholonautas.edu.pe.

GHIARDO, FeliPE (2004): «Generaciones y juventud: una relectura desde Mannheim y Ortega y Gasset». Última Década ํ⒛ Valparaíso: Ediciones CIDPA.

HOYL, M. TRINIDAD (2002): «Envejecimiento biológico». En: VV.AA.: $M a-$ nual de geriatría y gerontología. Santiago: Ediciones PUC.

IÁÑEZ PAREJA, ENRIQUE: «Introducción a la biotecnología». En: www.ugr.es.

LACADENA, JUAN RAMÓN (a): «El genoma: esperanza y amenaza». En: www.diariomedico.com.

— (b): «El proyecto genoma humano: consideraciones éticas». En: www.cnice.mecd.es.

MANNHEIM, KARL (1990): Le problème des générations. Paris: Nathan.

MARTÍN CRIADO, ENRIQUE (a): «Generaciones/clases de edad». En: www.ucm.es.

(b): «Habitus». En: www.ucm.es.

PEREIRA GONZÁLEZ, LUZ MARINA: «La inmortalidad: ¿un salto al vacío?». En: www.galeon.com.

RECOLETOS GRUPO DE COMUNICACIÓN: www.ondasalud.com.

WALLON, HENRI, JEAN PIAGET y ERIK ERIKSON: «El desarrollo e involución de la conducta». En: www.gestiopolis.com. 


\section{Cuadro 1}

Agentes y subagentes en el campo de la edad

\begin{tabular}{|c|c|c|c|}
\hline SUBCAMPO & AGENTE & SUBAGENTE & APUESTAS O PRÁCTICAS \\
\hline \multirow{4}{*}{ Longevidad } & Estado & $\begin{array}{l}\text { Ministerios (educa- } \\
\text { ción, salud, etc.), } \\
\text { municipios, aparato } \\
\text { jurídico/legislativo, } \\
\text { servicios. }\end{array}$ & $\begin{array}{c}\text { Políticas y programas destinados a la } \\
\text { protección social, el cuidado de la salud y } \\
\text { del medioambiente; la introducción y } \\
\text { sostenimiento de estilos de vida saludables. } \\
\text { Estímulos a la investigación de la ciencia } \\
\text { biomédica. }\end{array}$ \\
\hline & Mercado & \begin{tabular}{|c|} 
Laboratorios e indus- \\
trias farmacéuticas. \\
Empresas en el campo \\
de la medicina y/o ane- \\
xas al cuidado de la
\end{tabular} & $\begin{array}{l}\text { Investigación en el campo de la genética y } \\
\text { de las biotecnologías. } \\
\text { Intervenciones en cirugía estética; trata- } \\
\text { mientos de la piel, salud bucal. }\end{array}$ \\
\hline & & $\begin{array}{c}\text { piel } \\
\text { Industria del deporte o } \\
\text { vinculadas al físico } \\
\text { culturismo (gimnasios, } \\
\text { termas). } \\
\text { Industria alimentaria }\end{array}$ & $\begin{array}{l}\text { Venta de máquinas, promociones turísti- } \\
\text { cas, planes de mantención física, etc. } \\
\text { Cultivo prácticas de alimentación «sanas». }\end{array}$ \\
\hline & $\begin{array}{l}\text { Sociedad } \\
\text { Civil }\end{array}$ & $\begin{array}{l}\text { Institutos, centros, } \\
\text { organizaciones. }\end{array}$ & $\begin{array}{l}\text { Cultivo de prácticas de la salud, de filoso- } \\
\text { fías y de estilos de vida alternativos. } \\
\text { Promoción de cuidado del medioambiente. }\end{array}$ \\
\hline \multirow{3}{*}{$\begin{array}{l}\text { Clases } \\
\text { de edad }\end{array}$} & Estado & \begin{tabular}{|l|} 
Ministerios, munici- \\
pios, aparato jurídi- \\
co/legislativo, servi- \\
cios de nivel nacional. \\
\end{tabular} & $\begin{array}{l}\text { Políticas y programas sectoriales etarios. } \\
\text { Regulaciones de prácticas productivas, } \\
\text { derechos, deberes, con criterios de edad. }\end{array}$ \\
\hline & Mercado & \begin{tabular}{|} 
Industria cultural, \\
industria vestimentaria, \\
alimentaría, de la \\
sociabilidad. \\
Industria publicitaria y \\
de medios de comuni- \\
cación. Mercadotecnia.
\end{tabular} & $\begin{array}{l}\text { Promoción de prácticas y de estilos de vida } \\
\text { vinculados a clases de edad. }\end{array}$ \\
\hline & $\begin{array}{l}\text { Sociedad } \\
\text { Civil }\end{array}$ & $\begin{array}{l}\text { Institutos, centros, } \\
\text { organizaciones. }\end{array}$ & $\begin{array}{l}\text { Promoción de prácticas que obedecen a } \\
\text { criterios de clases de edad (scoutismo, } \\
\text { beneficencia, voluntariado). }\end{array}$ \\
\hline \multirow{3}{*}{ Generaciones } & Estado & \begin{tabular}{|l|} 
Ministerios, munici- \\
pios, aparato jurídi- \\
co/legislativo, servi- \\
cios de nivel nacional. \\
\end{tabular} & $\begin{array}{l}\text { Cambios en las condiciones materiales y } \\
\text { culturales de la producción de los sujetos. }\end{array}$ \\
\hline & Mercado & $\begin{array}{l}\text { Industria cultural } \\
\text { Industria vestimenta- } \\
\text { ria, alimentaria, de la } \\
\text { sociabilidad. }\end{array}$ & $\begin{array}{l}\text { Cambios en las condiciones materiales y } \\
\text { culturales de la producción de los sujetos. }\end{array}$ \\
\hline & $\begin{array}{l}\text { Sociedad } \\
\text { Civil }\end{array}$ & $\begin{array}{c}\text { Industria publicitaria y } \\
\text { de medios de comuni- } \\
\text { cación, Mercadotecnia. } \\
\text { Generaciones cons- } \\
\text { truidas metodológica- } \\
\text { mente. }\end{array}$ & Habitus generacionales. \\
\hline
\end{tabular}


